\title{
Morphoproteomics demonstrates activation of mammalian target of rapamycin pathway in papillary thyroid carcinomas with nuclear translocation of MTOR in aggressive histological variants
}

\author{
Jing Liu and Robert E Brown
}

Department of Pathology and Laboratory Medicine, University of Texas Health Science Center at Houston Medical School, Houston, TX, USA

\begin{abstract}
We used morphoproteomics to investigate mammalian target of rapamycin (MTOR) signaling pathway in papillary thyroid carcinomas and correlated the results with clinicopathological parameters. Archival paraffinembedded tissue of papillary thyroid carcinomas was obtained from 30 patients, including 15 classical type and 8 follicular, 4 tall-cell, 1 columnar-cell, 1 diffuse sclerosing and 1 cribriform variants. Immunohistochemical stains were performed for three phosphorylated (p) protein analytes: p-MTOR (Ser2448), p-Akt (Ser473) and p-p70S6K (Thr389). Chromogenic signals and subcellular compartmentalization (nuclear, cytoplasmic and plasmalemmal) were evaluated. Clinicopathological parameters were reviewed. Immunoreactivities for p-MTOR, p-Akt and p-p70S6K were observed in all papillary thyroid carcinomas. In addition to an expression of p-MTOR in cytoplasmic location, nuclear translocation of p-MTOR with variable loss of plasmalemmal expression, and with concomitant nuclear expression of p-Akt, was also identified in all tall-cell, columnar-cell and diffuse sclerosing variants of papillary thyroid carcinoma. There were no significant differences in the clinicopathological parameters, including tumor size, extrathyroidal extension, angioinvasion and nodal metastases between the groups with and without nuclear expression of $p-M T O R(P>0.05)$. The expressions of $p-M T O R$ in cytoplasmic and/or plasmalemmal locations with the concomitant immunoreactivity for p-p70S6K in all papillary thyroid carcinomas indicate the activation of MTOR complex 1 pathway. The nuclear translocation of p-MTOR evidences the activation of MTOR complex 2 and is identified only in the known aggressive histological variants of papillary thyroid carcinoma, including tall-cell, columnar-cell and diffuse sclerosing variants. Thus, these results suggest the constitutive activation of MTOR signaling pathway in papillary thyroid carcinomas and provide a new insight of biogenetic basis for the aggressive histological variants of papillary thyroid carcinoma. The pattern of expression of p-MTOR in papillary thyroid carcinomas may serve as a diagnostic/ prognostic marker and a potential therapeutic target.

Modern Pathology (2011) 24, 1553-1559; doi:10.1038/modpathol.2011.121; published online 5 August 2011
\end{abstract}

Keywords: Akt; immunohistochemistry; mammalian target of rapamycin; morphoproteomics; papillary thyroid carcinoma

Papillary thyroid carcinoma is the most common type of thyroid malignant neoplasm. Most papillary

Correspondence: Dr J Liu, MD, PhD, Department of Pathology and Laboratory Medicine, University of Texas Health Science Center at Houston Medical School, 6431 Fannin Street, MSB 2.260A, Houston, TX 77030, USA.

E-mail: jing.liu.1@uth.tmc.edu

Received 10 February 2011; revised 18 May 2011; accepted 31 May 2011; published online 5 August 2011 thyroid carcinomas are indolent and have an excellent prognosis with appropriate treatment, including surgery and postoperative radioactive iodine ablation with follow-up monitoring. Overall survival is greater than $90 \%{ }^{1}$ In contrast to the overall good prognosis, a small number of papillary thyroid carcinomas present a more aggressive course. Some particular histological variants, including columnar-cell, tall-cell, solid and diffuse sclerosing variants, have been linked to unfavorable 
outcome. $^{2}$ The tall-cell, columnar-cell and diffuse sclerosing variants have a tumor-related mortality of $25 \%, 90 \%$ and $5.7 \%$, respectively, in comparison with $0.6 \%$ in conventional papillary thyroid carcinomas. ${ }^{3-6}$ Several prognostic clinicopathological parameters have been proposed to define low- and high-risk groups of patients with thyroid cancers. ${ }^{7-9}$ However, the difference in tumorigenesis of these aggressive variants from the majority of papillary thyroid carcinoma with an indolent behavior is unknown.

Recently, some biogenetic alterations, eg, oncogene BRAF, have been discovered in some papillary thyroid carcinomas, which may have diagnostic, prognostic and therapeutic implications. ${ }^{10,11}$ However, the results are controversial. ${ }^{12}$ Although the study of papillary thyroid carcinoma at molecular level has been focused on the RAS-RAF-MEK-ERKMAP kinase pathway, the majority of other signaling pathways possibly involved in the tumorigenesis of papillary thyroid carcinomas have not been fully investigated.

Among cancer signaling pathways, the mammalian target of rapamycin (MTOR) pathway has been increasingly studied and its activation has been reported in various types of malignancy. However, only a few studies of MTOR pathway in papillary thyroid carcinoma were published in the literature. ${ }^{13-16}$ These studies were limited to scattered genes and their products using cultured cells or animal models. Recently, several MTOR inhibitors, as potential pharmaceutical agents, have been identified and some of them have been utilized in clinical or preclinical studies for a number of malignancies. ${ }^{17-19}$ This advancement raises the question whether these agents can be used for treatment-resistant papillary thyroid carcinomas. Therefore, it becomes urgent to discover the role of MTOR pathway in papillary thyroid carcinomas.

The MTOR is a highly conserved protein kinase and a central controller of cell growth and metabolisms. ${ }^{20}$ It has an integral role in coordinating cell growth, cell proliferation, angiogenesis and metabolism in response to growth factors, nutrients and the energy status of the cells, and promotes cell cycle progression and suppresses apoptosis in a variety of human cancers. ${ }^{18,19,21}$ The MTOR is found in two structurally and functionally distinct multiprotein complexes, MTOR complex 1 (MTORC1) containing raptor and MTOR complex 2 (MTORC2) containing rictor. Each of the MTORC signals via a different set of effector pathways, ie, MTORC1 through p70S6K and MTORC2 through Akt. ${ }^{22,23}$ Collaborating in tumorigenesis, MTORC1 contributes to cell proliferation and MTORC2 is thought to mediate cell survival. Rapamycin is an MTOR inhibitor to prevent MTOR-dependent downstream signaling transduction. MTORC1 is rapamycin sensitive, whereas MTORC2 is relatively rapamycin insensitive. Therefore, it is crucial to identify the MTORC2 activation in tumors, and there may be other implications of the MTORC2 activation in addition to the different treatment responses. To our knowledge, the study of MTORC2 in papillary thyroid carcinomas has not been reported in the literature.

Morphoproteomics utilizes immunohistochemical staining methods to identify the expression of protein analytes in signal transduction pathways in lesional tissue. ${ }^{24}$ It relies on the use of phosphospecific probes directed against putative sites of activation of molecules, subcellular compartmentalization and/or correlative expressions of protein analytes to assess the constitutive activation of cell signaling pathways and to expose potential targets at molecular level for individualized therapy.

In this study, we investigated the MTOR signaling pathway, including its upstream regulators and downstream effectors, using morphoproteomics on patients' tissue of various types of papillary thyroid carcinoma and examined how clinicopathological parameters previously proposed correlated with the MTOR signaling pathway.

\section{Materials and methods}

Archival thyroidectomy specimens were obtained between 2001 and 2008 and from 30 patients with papillary thyroid carcinomas, including 15 classical type and 8 follicular, 4 tall-cell, 1 columnar-cell, 1 diffuse sclerosing and 1 cribriform variants. The protocol of this study was approved by the Institutional Review Board of the University of Texas Health Science Center at Houston.

\section{Immunohistochemical Staining}

Immunohistochemical stains were performed on formalin-fixed and paraffin-embedded unstained sections of $4 \mu \mathrm{m}$ thickness. Three primary antibodies were utilized for immunohistochemical staining to detect phosphorylated (p) antigens, including p-Akt (Ser473; Cell Signaling Technology, Beverly, MA, USA), p-MTOR (Ser2448; Cell Signaling Technology) and p-p70S6K (Thr389; Cell Signaling Technology). The unstained sections were deparaffinized in xylene and rehydrated in a graded series of ethanols. Heat-induced epitope retrieval was performed. A semiautomatic method and monoclonal antibodies were used against these antigens. Endogenous pigments were quenched with $3 \% \mathrm{H}_{2} \mathrm{O}_{2}$ in methanol for $10 \mathrm{~min}$ and rinsed with Tris-buffered saline with Tween 20. A few drops of diluted normal blocking serum were placed on the tissue and incubated at room temperature. The serum was blotted off and the slides were incubated with primary antibody overnight at $4^{\circ} \mathrm{C}$. The remaining procedure took place on a DAKO Autostainer programmed to treat each slide with diluted biotinylated secondary antibody for $30 \mathrm{~min}$. The slides were rinsed and incubated with 3,3'-diaminobenzidine chromogen solution (EnVision + System Kit, 
DAKO) for $10 \mathrm{~min}$. The slides were rinsed again and counterstained with Gill II hematoxylin, treated with xylene and coverslipped. Appropriate positive and negative controls for each case were obtained.

\section{Assessment of Immunohistochemical Staining}

Chromogenic signal and subcellular expression pattern were assessed for each marker by brightfield microscopy. The subcellular expression pattern was characterized as: nuclear, cytoplasmic or plasmalemmal locations.

\section{Histological Evaluation and Clinical/Radiological Correlation}

All pathology reports, hematoxylin and eosin (H\&E)stained sections and clinical documents, including clinical notes and radiology reports, were reviewed. The tall-cell variant of papillary thyroid carcinoma is defined as at least $30 \%$ tumor cells with a height to width ratio of $\geq 3$ and abundant eosinophilic cytoplasm besides the nuclear features as in classical papillary thyroid carcinoma.

\section{Statistical Analyses}

Fisher's exact test (two tailed) and Student's $t$-test (two tailed) were used to evaluate the differences in clinicopathological parameters of papillary thyroid carcinomas between the groups with and without nuclear expression of p-MTOR. The significance level was set at $P<0.05$.

\section{Results}

\section{Immunohistochemistry}

Immunoreactivities for p-MTOR (Ser2448), p-Akt (Ser473) and p-p70S6K (Thr389) were observed in all papillary thyroid carcinomas $(n=30)$. The expression of p-MTOR in cytoplasmic and/or plasmalemmal locations was variable $(1+$ to $3+$ in $50-100 \%$ of tumor cells). In addition, the nuclear translocations of p-MTOR $(1+$ to $2+$ in $50-100 \%$ of tumor cells) and p-Akt $(1+$ to $2+$ in $30-80 \%$ of tumor cells) were identified in all tall-cell, columnar-cell and diffuse sclerosing variants of papillary thyroid carcinoma. The nuclear translocation of p-MTOR (Ser2448) was accompanied by a loss of plasmalemmal expression in some areas of all aggressive histological variants of papillary thyroid carcinoma. There was no nuclear expression of p-MTOR in classical type and cribriform and follicular variants of papillary thyroid carcinoma. The expression of p-p70S6K was moderate to strong, extensive, and nuclear in location in all papillary thyroid carcinomas $(2+$ to $3+$ in $>95 \%$ of tumor nuclei). The expressions of these protein analytes are summarized in Table 1. Examples of $\mathrm{H} \& \mathrm{E}$ and immunostains for p-MTOR in classical and tall-cell variant of papillary thyroid carcinomas are demonstrated in Figure 1.

\section{Correlation of Clinicopathological Parameters and Nuclear Expression of p-MTOR}

The clinicopathological parameters of papillary thyroid carcinomas are summarized in Table 2. The sizes of the papillary thyroid carcinomas with and without nuclear expression of p-MTOR ranged from 0.9 to $6.2 \mathrm{~cm}$ (mean: $3.3 \mathrm{~cm}$ ) and 0.2 to 8.5 (mean: $2.5 \mathrm{~cm})$, respectively $(P=0.38)$. The numbers of cases with extrathyroidal extension, angioinvasion and nodal metastases are not significantly different between the groups with and without nuclear expression of p-MTOR. There were no distant metastases in any of the cases.

\section{Discussion}

Our study demonstrates: (1) constitutive activation of MTOR pathway in all types/variants of papillary thyroid carcinoma indicated by the expression and phosphorylation at putative sites, Ser2448 in MTOR, Ser473 in Akt and Thr389 in p70S6K; (2) nuclear translocation of p-MTOR, suggestive of overexpression/activation of MTORC2 in aggressive histological variants of papillary thyroid carcinoma; and (3) no significant differences in tumor size, extrathyroidal extension, angioinvasion and nodal metastases between the groups of papillary thyroid

Table 1 Expressions of p-Akt (Ser473), p-MTOR (Ser2448) and p-p70S6K (Thr389) in papillary thyroid carcinomas

\begin{tabular}{lcccccc}
\hline Protein analytes & $\begin{array}{c}\text { Staining } \\
\text { intensity }\end{array}$ & $\begin{array}{c}\text { Classical } \\
\text { type }\end{array}$ & $\begin{array}{c}\text { Follicular } \\
\text { variant }\end{array}$ & $\begin{array}{c}\text { Cribriform- } \\
\text { variant }\end{array}$ & $\begin{array}{c}\text { Tall-cell } \\
\text { variant }\end{array}$ & $\begin{array}{c}\text { Columnar- } \\
\text { cell } \\
\text { variant } \\
\text { valerosing } \\
\text { variant }\end{array}$ \\
\hline Nuclear p-Akt (Ser473) & $1-2+$ & $13 / 15$ & $7 / 8$ & $1 / 1$ & $4 / 4$ & $1 / 1$ \\
Cytoplasmic p-Akt (Ser473) & $1-2+$ & $15 / 15$ & $8 / 8$ & $1 / 1$ & $4 / 4$ & $1 / 1$ \\
Nuclear p-MTOR (Ser2448) & $1-2+$ & $0 / 15$ & $0 / 8$ & $0 / 1$ & $4 / 4$ & $1 / 1$ \\
Cytoplasmic p-MTOR (Ser2448) & $1-3+$ & $15 / 15$ & $8 / 8$ & $1 / 1$ & $4 / 4$ & $1 / 1$ \\
Plasmalemmal p-MTOR (Ser2448) & $1-3+$ & $15 / 15$ & $8 / 8$ & $1 / 1$ & $4 / 4$ & $1 / 1$ \\
Nuclear p-p70S6K (Thr389) & $2-3+$ & $15 / 15$ & $8 / 8$ & $1 / 1$ & $4 / 4$ & $1 / 1$ \\
\hline
\end{tabular}



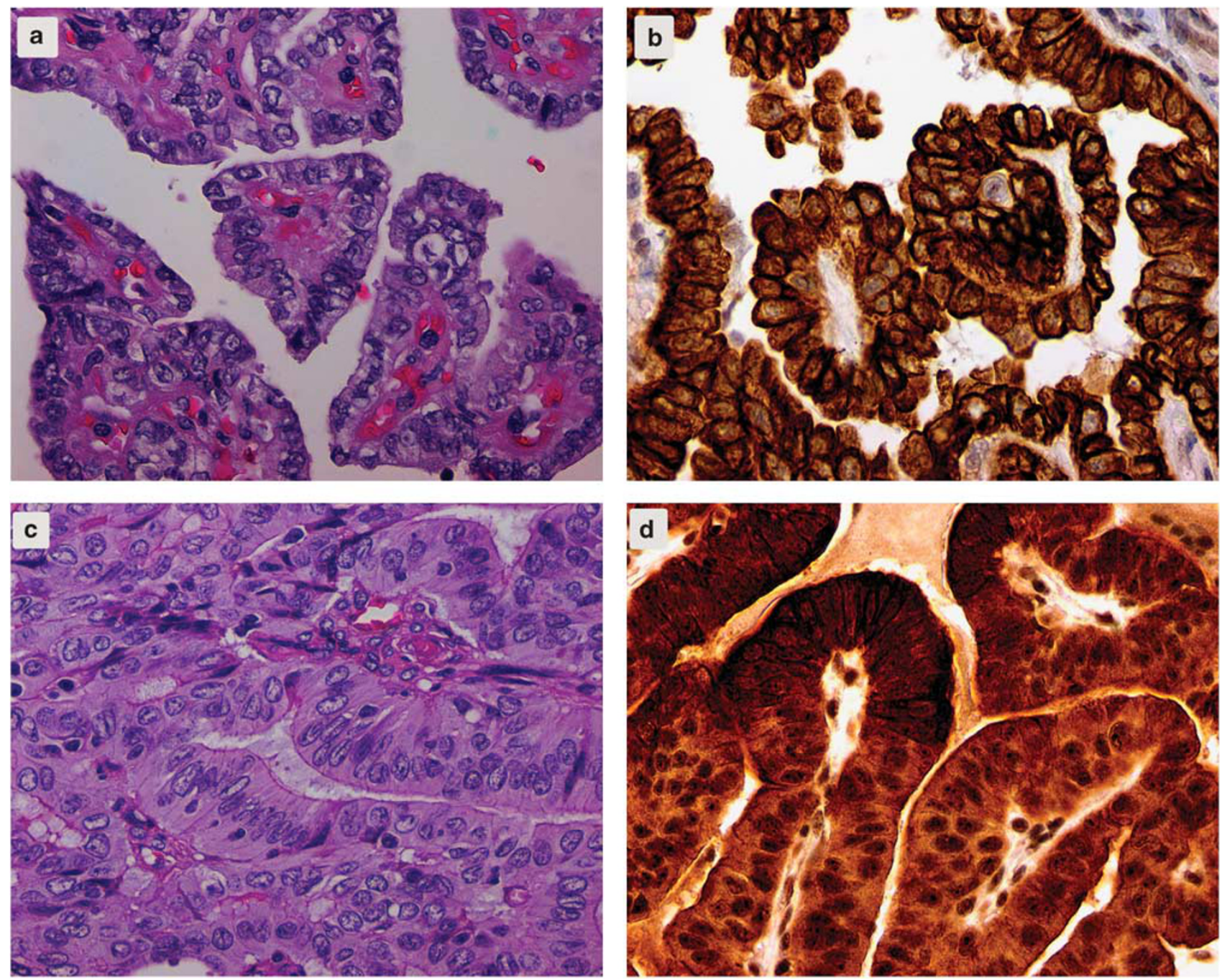

Figure 1 Classical type of papillary thyroid carcinoma: (a) hematoxylin and eosin $(\mathrm{H} \& \mathrm{E})$ stain $(\times 400)$ and $(\mathbf{b})$ immunohistochemial staining of phosphorylated mammalian target of rapamycin (p-MTOR; Ser2448) showing cytoplasmic and plasmalemmal positivity $(\times 400)$; and tall-cell variant of papillary thyroid carcinoma: $(\mathbf{c})$ H\&E stain $(\times 400)$ and $(\mathbf{d})$ immunohistochemial staining of p-MTOR (Ser2448) demonstrating nuclear and cytoplasmic reactivity with or without plasmalemmal expression $(\times 400)$.

Table 2 Summary of tumor size, extrathyroidal extension, angioinvasion and nodal metastases in nuclear p-MTOR (Ser2448)-positive and -negative papillary thyroid carcinomas

\begin{tabular}{|c|c|c|c|}
\hline Clinicopathological parameters & $\begin{array}{l}\text { Positive nuclear } \\
\text { p-MTOR }(\mathrm{n}=6)\end{array}$ & $\begin{array}{l}\text { Negative nuclear } \\
p \text {-MTOR }(\mathrm{n}=24)\end{array}$ & $\mathrm{P}$-value \\
\hline Tumor size (cm; $M \pm$ s.d.) & $3.32 \pm 2.15$ & $2.50 \pm 1.98$ & 0.38 \\
\hline Extrathyroidal extension (number of cases) & $3 / 6(50.0 \%)$ & $9 / 24(37.5 \%)$ & 0.66 \\
\hline Angioinvasion (number of cases) & $2 / 6(33.3 \%)$ & $6 / 24(25.0 \%)$ & 0.65 \\
\hline Nodal metastasis (number of cases) & $4 / 6(66.7 \%)$ & $9 / 24(37.5 \%)$ & 0.36 \\
\hline
\end{tabular}

carcinomas with and without nuclear expression of p-MTOR.

It has been demonstrated that the p-MTOR expressed in cytoplasm and/or plasmalemma indicates the activated state of MTOR in renal cell carcinoma, gastric adenocarcinoma and other tumors. ${ }^{25-27}$ The activated Akt promotes MTORC1 signaling by phosphorylating and inhibiting TSC1/ TSC2-negative regulatory complex. The MTORC1 phosphorylatively activates p70S6K at threonine 389 , one of its downstream effectors. ${ }^{22,27,28}$ The concomitant expression of p-Akt, p-MTOR and p-p70S6K demonstrated in the current study is consistent with the activation of MTORC1 signaling 
pathway in papillary thyroid carcinomas. The activation status is further evidenced by the nuclear translocation of p-p70S6K. ${ }^{29}$ In support of our observation of the activation of Akt-MTORC1p70S6K pathway in papillary thyroid carcinomas is a recent in vitro study showing potent inhibition of cell proliferation by perifosine, an Akt inhibitor, and temsirolimus, an MTORC1 inhibitor, in cultured papillary thyroid carcinoma cells of TPC1 cell line that harbored PI3K/Akt-activating genetic alterations. ${ }^{16}$

Moreover, the current study shows a shift in the subcellular compartmentalization of p-MTOR (Ser2448). Specifically, there is a loss of predominantly plasmalemmal expression coupled with increased nuclear signal intensity of p-MTOR in some areas of the aggressive histological variants of papillary thyroid carcinoma. In an in vitro study, Rosner and Hengstschlager found that MTORC1 assembly occurred predominantly in the cytoplasm, whereas MTORC2 is abundant in both cytoplasm and nucleus of non-transformed, non-immortalized human diploid fibroblasts. ${ }^{30}$ Such findings imply that the expression of MTOR in the nuclear location is attributed to the presence of MTORC2. Accordingly, our results indicate that there is constitutive activation of MTORC2 in the aggressive variants of papillary thyroid carcinoma, which may imply that there are some differences in tumorigenesis of the aggressive histological variants from the classical type and other less aggressive variants of papillary thyroid carcinoma.

One of our previous studies showed strong nuclear expression $(3+)$ of p-MTOR in anaplastic thyroid carcinomas and no nuclear expression in normal thyroid tissue. ${ }^{31}$ In comparison, the nuclear positivity of the p-MTOR in anaplastic thyroid carcinoma is stronger than the $1+$ to $2+$ signal intensity seen in tall-cell, columnar-cell and diffuse sclerosing variants of papillary thyroid carcinoma in the current study. On the basis of these observations, we feel that the staining intensity of MTORC2 increases in a continuous spectrum, which correlates with the degree of aggressiveness of the thyroid cancers.

Furthermore, Akt has been identified to be the downstream effector of MTORC2 by recent studies. $^{32,33}$ Phosphorylation of two sites on Akt activates the Akt: one at Thr308 by PDK1 and the other at Ser473 mainly by MTORC2 in both nuclear and cytoplasmic compartments. ${ }^{23,33,34}$ These phosphorylation events can occur independently or interactively. Although phosphorylation of Akt at Thr308 alone empowers Akt with enough activity to phosphorylate many of its substrates, maximal Akt activity requires phosphorylation at both Thr308 and Ser473. It has been postulated that the phosphorylation of Akt at Ser473 stimulates Akt phosphorylation at Thr308 by PDK1 so that Ser473 is a critical regulatory site required for maximal Akt kinase activity. ${ }^{33,35}$ Our study demonstrates the expression of p-Akt (Ser473), consistent with the downstream effect of MTORC2 activation in addition to being an activated upstream regulator of MTORC1.

However, the expression of p-Akt in the current study is only modest to moderate but not strong, which may be related to the interaction among Akt, MTORC1 and MTORC2. A few previous studies suggested that after Akt activated MTORC1, the latter elicited negative feedback loops to inhibit Akt activity through p70S6K and insulin receptor substrate $1 .^{34}$ Therefore, Akt is placed under positive and negative control mediated by the MTORCs. The lower level of expression of p-Akt in this study than that expected is similar to that noted in our previous study on anaplastic thyroid carcinoma and is thought to be due to the negative feedback loop elicited by MTORC1. ${ }^{31}$ In accord, rapamycin resistance is noted in many types of tumors, as rapamycin and its analogs, as the first class of MTOR inhibitors, have been identified and utilized in clinical or preclinical studies for malignancies, which is attributable to the MTORC1-dependent negative feedback loops. ${ }^{36-38}$

The activation of MTORC2/Akt pathway, conferring rapamycin resistance, has been reported in some malignant neoplasms. ${ }^{34}$ Therefore, our observation of the activation of the MTORC2/Akt pathway has therapeutic implications.

The current study does not show significant differences in tumor size, extrathyroidal extension, angioinvasion and nodal metastases between the groups of papillary thyroid carcinoma with and without nuclear expression of p-MTOR. These clinicopathological parameters have been known to be of value in papillary thyroid carcinoma prognosis. However, the conclusions derived from previous retrospective studies were often limited by uncontrolled variation in the severity of the initial disease, ie, that the more severe initial disease (large tumor size, extrathyroidal extension, angioinvasion and metastases) has the worse prognosis. With the widespread use of ultrasound examination and fine-needle aspiration, more papillary thyroid carcinomas have been detected and surgically treated at an earlier stage than ever. In the current study, there were trends toward increased tumor size, extrathyroidal extension, angioinvasion and nodal metastases in the papillary thyroid carcinomas with nuclear expression of p-MTOR, although not statistically significant, which might be due to the fact that our data had been only partially influenced by the utility of ultrasound examination and fine-needle aspiration for evaluating thyroid lesions in recent years. A larger series of study of papillary thyroid carcinomas with modern diagnostic modalities is desired to elucidate clinicopathological parameters for predicting the papillary thyroid carcinoma prognosis.

In summary, this study demonstrates immunoreactivities for $\mathrm{p}-\mathrm{MTOR}$, p-Akt and p-p70S6K in all 
papillary thyroid carcinomas and nuclear translocation of p-MTOR in tall-cell, columnar-cell and diffuse sclerosing variants. The findings indicate constitutive activation of the MTOR pathway in all papillary thyroid carcinomas and evidence MTORC2 activation in the aggressive histological variants of papillary thyroid carcinoma. The nuclear expression of p-MTOR is not correlated with tumor size, extrathyroidal extension, angioinvasion and nodal metastases. Such findings may be of diagnostic, prognostic and, most importantly, therapeutic value.

\section{Acknowledgements}

We thank Ms Pamela K Johnston, HT (ASCP) for her technical assistance and Ms Bheravi Patel for secretarial and graphic design expertise.

\section{Disclosure/conflict of interest}

The authors declare no conflict of interest.

\section{References}

1 Livolsi VA, Albores-Saavedra J, Asa SL, et al. Papillary carcinoma In: DeLellis RA, Lloyd RV, Heitz PU, Eng C (eds). WHO classification of tumors - pathology and genetics of tumours of endocrine organs. IARC press: Lyon, France, 2004, pp 57-66.

2 Nikiforov YE, Ohori NP. Papillary carcinoma In: Nikiforov YE, Biddinger PW, Thompson LDR (eds). Diagnostic pathology and molecular genetics of the thyroid. Wolters Kluwer/Lippincott William \& Wilkins: Philadelphia, Pennsylvania, 2009, pp 160-213.

3 Johnson TL, Lloyd RV, Thompson NW, et al. Prognostic implications of the tall cell variant of papillary thyroid carcinoma. Am J Surg Pathol 1988;12:22-27.

4 Evans HL. Columnar-cell carcinoma of the thyroid. A report of two cases of an aggressive variant of thyroid carcinoma. Am J Clin Pathol 1986;85:77-80.

5 Fukushima M, Ito Y, Hirokawa M, et al. Clinicopathologic characteristics and prognosis of diffuse sclerosing variant of papillary thyroid carcinoma in Japan: an 18year experience at a single institution. World J Surg 2009;33:958-962.

6 Falvo L, Giacomelli L, D’Andrea V, et al. Prognostic importance of sclerosing variant in papillary thyroid carcinoma. Am Surg 2006;72:438-444.

7 Hay ID, Grant CS, Taylor WF, et al. Ipsilateral versus bilateral lobar resection in papillary thyroid carcinoma: a retrospective analysis of surgical outcome using a novel prognostic scoring system. Surgery 1987;102:1088-1095.

8 Byar DP, Green SB, Dor P, et al. A prognostic index for thyroid carcinoma. A study of the EORTC thyroid cancer cooperative group. Eur J Cancer 1979; 15:1033-1041.

9 Cady B, Rossi R. An expanded view of risk-group definition in differentiated thyroid carcinoma. Surgery 1988;104:947-953.
10 Smith RA, Salajegheh A, Weinstein S, et al. Correlation between BRAF mutation and the clinicopathological parameters in papillary thyroid carcinoma with particular reference to follicular variant. Hum Pathol 2010;42:500-506.

11 Lloyd RV, Buehler D, Khanafshar E. Papillary thyroid carcinoma variants. Head Neck Pathol 2011;5: 51-56.

12 Tang KT, Lee CH. BRAF mutation in papillary thyroid carcinoma: pathogenic role and clinical implications. J Chin Med Assoc 2010;73:113-128.

13 Hou P, Bojdani E, Xing M. Induction of thyroid gene expression and radioiodine uptake in thyroid cancer cells by targeting major signaling pathways. J Clin Endocrinol Metab 2010;95:820-828.

14 Jin N, Jiang T, Rosen DM, et al. Dual inhibition of mitogen-activated protein kinase kinase and mammalian target of rapamycin in differentiated and anaplastic thyroid cancer. J Clin Endocrinol Metab 2009; 94:4107-4112.

15 Rocha AS, Paternot S, Coulonval K, et al. Cyclic AMP inhibits the proliferation of thyroid carcinoma cell lines through regulation of CDK4 phosphorylation. Mol Biol Cell 2008;19:4814-4825.

16 Liu D, Hou P, Liu Z, et al. Genetic alterations in the phosphoinositide 3-kinase/Akt signaling pathway confer sensitivity of thyroid cancer cells to therapeutic targeting of Akt and mammalian target of rapamycin. Cancer Res 2009;69:7311-7319.

17 Galanis E, Buckner JC, Maurer MJ, et al. Phase II trial of temsirolimus (CCI-779) in recurrent glioblastoma multiforme: a north central cancer treatment group study. J Clin Oncol 2005;23:5294-5304.

18 Agarwala SS, Case S. Everolimus (RAD001) in the treatment of advanced renal cell carcinoma: a review. Oncologist 2010;15:236-245.

19 Antonarakis ES, Carducci MA, Eisenberger MA. Novel targeted therapeutics for metastatic castration-resistant prostate cancer. Cancer Lett 2010;291:1-13.

20 Wullschleger S, Loewith R, Hall MN. TOR signaling in growth and metabolism. Cell 2006;124:471-484.

21 Guerin DA, Sabatini DM. Defining the role of mTOR in cancer. Cancer Cell 2007;12:9-22.

22 Nojima H, Tokunaga C, Eguchi S, et al. The mammalian target of rapamycin (mTOR) partner, raptor, binds the mTOR substrates p70 S6 kinase and 4E-BP1 through their TOR signaling (TOR) motif. J Biol Chem 2003;278:15461-15464.

23 Bhaskar PT, Hay N. The two TORCs and Akt. Dev Cell 2007;12:487-502.

24 Brown RE. Morphoproteomics: exposing protein circuitries in tumors to identify potential therapeutic targets in cancer patients. Expert Rev Proteomics 2005; 2:337-348

25 Lin F, Zhang PL, Yang XJ, et al. Morphoproteomic and molecular concomitants of an overexpressed and activated mTOR pathway in renal cell carcinomas. Ann Clin Lab Sci 2006;36:283-293.

26 Feng W, Brown RE, Trung CD, et al. Morphoproteomic profile of mTOR, ras/raf kinase/ERK, and NF- $\kappa \mathrm{B}$ pathways in human gastric adenocarcinoma. Ann Clin Lab Sci 2008;38:195-209.

27 Brown RE, Zhang PL, Lun M, et al. Morphoproteomic and pharmacoproteomic rationale for mTOR effectors as therapeutic targets in head and neck squamous cell carcinoma. Ann Clin Lab Sci 2006;36: 273-282. 
28 Cao X, Kambe F, Moeller LC, et al. Thyroid hormone induces rapid activation of Akt/protein kinase B-mammalian target of rapamycin-p70S6K cascade through phosphatidylinositol 3-kinase in human fibroblasts. Mol Endocrinol 2005;19:102-112.

29 Reinhard C, Fernandez A, Lamb NJC, et al. Nuclear localization of p85s6k: functional requirement for entry into S phase. EMBO J 1994;13:1557-1565.

30 Rosner M, Hengstschlager M. Cytoplasmic and nuclear distribution of the protein complexes mTORC1 and mTORC2: rapamycin triggers dephosphorylation and delocalization of the mTORC2 components rictor and sin1. Hum Mol Genet 2008;17:2934-2948.

31 Liu J, Brown RE. Morphoproteomics demonstrates activation of mTOR pathway in anaplastic thyroid carcinoma: a preliminary observation. Ann Clin Lab Sci. 2010;40:211-217.

32 Hall MN. mTOR - what does it do? Transplant Proc 2008;40(10 suppl):S5-S8.
33 Sarbassov DD, Guertin DA, Ali SM, et al. Phosphorylation and regulation of Akt/PKB by the rector-mTOR complex. Science 2005;307:1098-1101.

34 Sparks CA, Guertin DA. Targeting mTOR: prospects for mTOR complex 2 inhibitors in cancer therapy. Oncogene 2010;29:3733-3744.

35 Stephens L, Anderson K, Stokoe D, et al. Protein kinase B kinases that mediate phosphatidylinositol 3,4,5-trisphosphate-dependent activation of protein kinase B. Science 1998;279:710-714.

36 Guertin DA, Sabatini DM. Defining the role of mTOR in cancer. Cancer Cell 2007;12:9-22.

37 Shor B, Gibbons JJ, Abraham RT, et al. Targeting mTOR globally in cancer: thinking beyond rapamycin. Cell Cycle 2009;8:3831-3837.

38 Borders EB, Bivona C, Medina PJ. Mammalian target of rapamycin: biological function and target for novel anticancer agents. Am J Health Syst Pharm 2010;67: 2095-2106. 